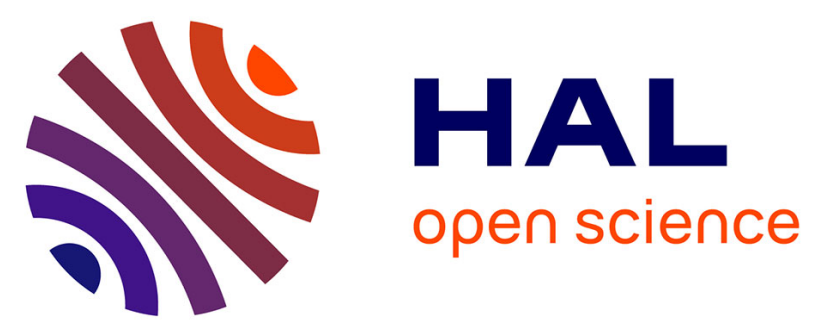

\title{
Prognosis of Streptococcus pneumoniae endocarditis in France, a multicenter observational study (2000-2015)
} Amandine Périer, Mathieu Puyade, Matthieu Revest, Pierre Tattevin, Louis Bernard, Adrien Lemaignen, David Boutoille, Joseph Allal, France Roblot, Blandine Rammaert

\section{To cite this version:}

Amandine Périer, Mathieu Puyade, Matthieu Revest, Pierre Tattevin, Louis Bernard, et al.. Prognosis of Streptococcus pneumoniae endocarditis in France, a multicenter observational study (20002015). International Journal of Cardiology, 2019, 288, pp.102-106. 10.1016/j.ijcard.2019.04.048 . hal-02534973

\section{HAL Id: hal-02534973 \\ https://hal.science/hal-02534973}

Submitted on 22 Oct 2021

HAL is a multi-disciplinary open access archive for the deposit and dissemination of scientific research documents, whether they are published or not. The documents may come from teaching and research institutions in France or abroad, or from public or private research centers.
L'archive ouverte pluridisciplinaire HAL, est destinée au dépôt et à la diffusion de documents scientifiques de niveau recherche, publiés ou non, émanant des établissements d'enseignement et de recherche français ou étrangers, des laboratoires publics ou privés.

\section{(ㅇ)(1) $\$$}

Distributed under a Creative Commons Attribution - NonCommerciall 4.0 International 
Prognosis of Streptococcus pneumoniae endocarditis in France, a multicenter observational study (2000-2015)

\section{Authors}

Amandine Périer ${ }^{1}$, Mathieu Puyade ${ }^{1}$, Matthieu Revest ${ }^{2}$, Pierre Tattevin ${ }^{2}$, Louis Bernard ${ }^{3}$, Adrien Lemaignen ${ }^{3}$, David Boutoille ${ }^{4}$, Joseph Allal ${ }^{5}$, France Roblot ${ }^{6}$, Blandine Rammaert $^{6}$

\section{Authors' affiliations}

${ }^{1}$ Université de Poitiers, Poitiers, France; CHU de Poitiers, Service de Médecine Interne, Poitiers, France

2 Université de Rennes, Rennes, France; CHU de Pontchaillou, Service de Maladies Infectieuses, Rennes, France

${ }^{3}$ Université de Tours, Tours, France; CHRU de Tours, Hôpital Bretonneau, Service de Médecine Interne et Maladies Infectieuses, Tours, France

${ }^{4}$ Université de Nantes, Nantes, France; CHU de Nantes, Service de Maladies Infectieuses, Nantes, France

${ }^{5}$ Université de Poitiers, Poitiers, France; CHU de Poitiers, Service de Cardiologie, Poitiers, France

${ }^{6}$ Université de Poitiers, Poitiers, France; CHU de Poitiers, Service de maladies infectieuses et tropicales, Poitiers, France; Inserm U1070, Poitiers, France

\section{Authorship}

The authors take responsibility for all aspects of the reliability and freedom from bias of the data presented and their discussed interpretation. 


\section{Corresponding author}

Dr Blandine Rammaert

Service de maladies infectieuses et tropicales

CHU de Poitiers

2 rue de la Milétrie

CS 90577

86021 Poitiers cedex

blandine.rammaert.paltrie@univ-poitiers.fr

tel : +33 549444422

fax : +33549444383

\section{Acknowledgment}

We thank Jeffrey Arsham for editing the manuscript

\section{Conflict of interest}

BR received travel grants for conferences from Pfizer, Gilead, Astellas, and speaker's fees from Merck/MSD, Gilead, and Basilea.

AL received travel grants for conferences from Pfizer and MSD and speaker's fees from MSD The other authors declare no conflict of interest.

\section{Keywords}

Infective endocarditis; Streptococcus pneumoniae; pneumococcus vaccination; prognosis 


\section{Introduction}

Infective endocarditis (IE) is an uncommon infection affecting 33.8 cases/million inhabitants/year in France (95\%CI, 30.8-36.9) [1]. Despite marked improvement in IE management, mortality can be as high as $20 \%$ [1]. IE due to Streptococcus spp. has declined and Staphylococcus aureus has become the first cause of this disease [1,2]. Streptococcus pneumoniae is currently responsible for less than $2 \%$ of IE cases [1]. However, only few studies on pneumococcal IE have been published over the last decade [3-5]. The other studies were published more than 10 years ago, and reported data from the 1990s [6-9].

Pneumococcal vaccination has significantly improved the prevention of invasive pneumococcal disease (IPD) over the last 15 years [10]. The European Commission granted marketing authorization valid throughout the European Union for 13-valent pneumococcal conjugate vaccine (PCV13) in 2009. In France, pneumococcal conjugate vaccines were initially recommended only in children. Albeit less effective, the 23-valent polysaccharide vaccine (PPV23) was the recommended vaccine in France for adults at risk of IPD from 1981 to 2017. Due to vaccination, IPD in adults decreased by $31 \%$ between 2009 and 2013 according to the French observatory network of pneumococcus [11]. Pneumonia is still the main clinical feature of IPD, accounting for $90 \%$ of cases, followed by meningitis (5\%), and other infections (5\%) such as endocarditis, septic arthritis, pleurisy and isolated bacteremia [12,13]. IPD has a high mortality rate: $20 \%$ in bacteremia and $30 \%$ in meningitis [14]. IPD has been linked with age, and with other factors such as COPD, alcoholism, smoking, heart failure, malignancies, and AIDS [14,15].

The aim of this study was to evaluate the prognosis of pneumococcal IE, and to determine clinical features and management of this infrequent IE. 


\section{Patients and Methods}

Study design. This retrospective observational study was conducted at four French university hospitals (Rennes, Nantes, Tours, Poitiers) from 1st January 2000 through 31st December 2015.

Ethics. The study was conducted in accordance with the Declaration of Helsinki. The National Data Protection Authority (Commission Nationale Informatique et Libertés), which is responsible for protection of individual data in France, approved the panel and its procedures.

Participants. All adult patients $\geq 18$ years old hospitalized for definite pneumococcal IE according to the modified Duke criteria were included [16]. Patients who developed IE due to S. pneumoniae were identified using ICD10: CA (I33.0) from the hospital computerized database.

The following data were retrospectively collected from medical records: sex, date of birth, date of first symptoms/diagnosis/surgery, risk factors for IPD and for IE, history of heart disease (heart valve localization, septic embolisms, echocardiographic and microbiological data, medical and surgical treatment), pneumococcus vaccination status, and outcome at 90 days and 2 years after diagnosis. In order to collect vaccination status and outcome at two years after diagnosis, general practitioners in charge of patients were contacted by telephone.

Definitions. IE risk factors included valvular prosthesis or intracardiac device, intravenous drug use, previous IE, congenital heart disease, valvular disease, aortic bicuspidy, hypertrophic obstructive cardiopathy and at-risk invasive procedure. An at-risk procedure was defined according to the 2015 European Society of Cardiology (ESC) guidelines [16]. 
Pneumococcal risk factors were defined as functional or anatomic asplenia, chronic pulmonary disease, repeated Ear-Nose-Throat (ENT) infections, chronic cardiopathy, alcohol abuse or chronic liver disease, AIDS, malignant blood disease, renal failure, transplantation, or age $\geq 65$ years.

Vascular lesions were defined as major arterial embolism, septic pulmonary infarct, infectious (mycotic) aneurysm, intracranial hemorrhage, conjunctival hemorrhage, or Janeway's lesion [16]. Immunological phenomena were defined according to the modified Dukes criteria as glomerulonephritis, Osler's nodes, Roth's spots, or rheumatoid factor [16].

Statistical analysis. Overall survival was measured from diagnosis to death from any cause, and IE survival was measured from diagnosis to IE-related death. Causes of death were independently reviewed by two investigators and classified as IE-related, or non IE-related. Overall survival was estimated using the Kaplan-Meier method. Censoring occurred for patients lost to follow-up. Quantitative variables were described as mean \pm standard deviation (SD) in the case of normal distribution or median and interquartile range [IQR 25-75] in the other cases. Comparisons were performed with Student T test if variances were equal or else with a non-parametric test (Mann Whitney). Qualitative variables were described as percentages, compared with Chi square test. Univariate analysis was performed for each of the selected criteria. Multivariate analysis was planned but not performed due to low number of events. Collinearity between variables was sought. A significance threshold for all analyses was defined at 0.05. All analyses were performed using SAS version 9.4 (SAS Institute Inc, Cary, North Carolina).

\section{Results}

\section{Demographic characteristics}


Among 3,886 definite IE hospitalized during the 15-year period of the study, 50 patients with pneumococcal IE were enrolled. The estimated frequency of pneumococcal IE was $1.3 \%$. Most of the patients included were males $(n=38 ; 76 \%)$ with a mean age of $60 \pm 14$ years at diagnosis. Demographic and clinical characteristics are shown in Table 1. IPD predisposing conditions were present in 39 patients (78\%), including alcohol abuse (34\%), chronic pulmonary disease (24\%), and age $\geq 65(36 \%)$. More than half of patients $(n=27 ; 54 \%)$ had at least two predisposing factors for IPD. Pneumococcal vaccination with 23-valent pneumococcal polysaccharide vaccine was performed before IE diagnosis in only two patients ( 6 and 3 years before IE, respectively). Of note, $76 \%$ of the patients had no risk factor for IE. The major IE risk factor was a previous valvular disease $(n=10,22 \%)$. Nine patients $(18 \%)$ had an intravascular device, either pacemaker $(n=4)$ or vascular prosthesis $(n=5)$. No patient had obstructive cardiopathy, aortic bicuspidy, AIDS or benefited from a previously at-risk procedure.

\section{Clinical characteristics}

The most prevalent clinical manifestation was fever (89\%). At least one vascular lesion was found in 10 patients (22\%). Concomitant foci of infection due to S. pneumoniae were present in 46 patients (92\%): pneumonia (48\%), meningitis (41\%), and arthritis (20\%), being the most common localizations (Table 1). Complete Austrian syndrome, which associated pneumococcal pneumonia, meningitis and endocarditis, was present in eight patients (16\%). None of these patients were older than 65 compare to 18 (43\%) patients in the group without Austrian syndrome. The group with Austrian syndrome had more alcohol abuse $(n=6 / 8 ; 75 \%)$ than the one without Austrian syndrome $(\mathrm{n}=11 / 42 ; 26 \% ; \mathrm{p}=0.009)$. All patients had TTE showing vegetations $(88 \%)$, valve regurgitation $(79 \%)$ or abscesses $(30 \%)$. Transesophageal echocardiogram was performed in 35 patients $(70 \%)$, heart CT in 4 patients (8\%), and no 
patient had cardiac positron emission tomography (PET)/CT. None of the patients with vascular prosthesis had dehiscence of the prosthesis. The aortic valve was more commonly damaged $(n=34,68 \%)$ than the mitral or tricuspid ones.

\section{Treatment}

The antimicrobial strategy was in accordance with the 2015 ESC Guidelines for antibiotic regimen in $74 \%$ and duration in $36 \%$ of the cases (Table 2$)$. Only $28 \%(n=14)$ received medical treatment fully in accordance with the 2015 ESC Guidelines. All patients received third-generation cephalosporins, amoxicillin or vancomycin. Seven patients (14\%) received a single antibiotic strategy, whereas gentamicin was combined with beta-lactams or glycopeptides in 39 patients (78\%). The combination with gentamicin was used for a shorter duration (8.5 days \pm 8.4 ) than the recommended two weeks. Patients who received combined therapy with beta-lactams but without gentamicin received vancomycin $(n=1)$, rifampicin $(n=2)$, or levofloxacin $(n=1)$. Overall antimicrobial treatment duration was two weeks longer than the recommended four weeks. For the five prosthetic valve endocarditis cases, the recommended six-week treatment was administered just once because of early mortality, and only this patient had surgery combined with medical treatment. Cardiac surgery was performed in 28 patients $(56 \%)$, in most cases $(n=25 ; 89 \%)$ through valve replacement (Table 2). A total of 22 patients (44\%) did not undergo surgery since they were not suitable for surgery $(n=5)$, or died early $(n=3)$, or had no surgical indication $(n=14)$. There was no significant difference in term of antibiotherapy between non-surgical patients who died and survived. Delay from IE diagnosis to cardiac surgery was 6.5 [2.0-10.5] days. In univariate analysis, patients who underwent surgery had fewer IPD risk factors $(\mathrm{p}=0.007)$ and chronic pulmonary disease ( 3 vs $9 ; \mathrm{p}=0.035)$, and they were significantly younger ( $56 \pm 13$ vs $65 \pm 14$ years; $p=0.010)$ than the medically treated patients (Supplemental Table 1). 


\section{Outcome}

Ten patients were lost to follow-up before 90 days. The 90-day survival rate was $83 \%(n=33)$

(Figure 1A). Among the seven deaths, six were due to IE complications (3 multi-visceral shock, 2 heart failure, 1 stroke) and one to pancreatic neoplasia. Among the five patients with pneumococcal prosthetic valve endocarditis, three died (1 multi-visceral shock, 1 heart failure, 1 stroke) within 60 days after diagnosis (22.0 days, [21.0-40.5]).

At 2 years, the survival rate was $67 \%(n=28)$ (Figure 1B). There were no more patients lost to follow-up. Four of the five additional deaths reported between 90 days and 2 years were due to neoplasia. The reason for the fifth death was unknown. At 2 years, the leading cause of death remained IE complications $(50 \%, n=6 / 12)$ even though these deaths occurred within the 90 days after diagnosis. The second mortality cause at 2 years was neoplasia $(42 \%, n=5 / 12)$. The risk factors for mortality at 2 years identified in the univariate analysis were age $>65$ (11 vs 4; $\mathrm{p}=0.011)$ and absence of vascular phenomena $(1$ vs $7 ; \mathrm{p}=0.048)$. By contrast, a surgical procedure was significantly associated with increased survival at 2 years $(15$ vs $6 ; \mathrm{p}=0.012)$. Kaplan Meier survival analysis did not show any significant difference between patients with and without meningitis. Regarding patients with Austrian syndrome, there were no more fatal issues $(\mathrm{p}=0.240)$ or relapses $(\mathrm{p}=0.204)$ compare to patients without Austrian syndrome.

Among the 50 patients, two had a relapse of pneumococcal IE. Although they were treated surgically, relapses occurred 48 hours and 6 months after the end of therapy, respectively. In each case, the damaged valve was different from the initial infected valve. The patient who relapsed at 6 months was vaccinated after IE diagnosis. A total of 13 patients were vaccinated with PPV23 after IE diagnosis. 


\section{Discussion}

To the best of our knowledge, our study is the largest homogeneous cohort of pneumococcal IE since 2000. It underscored that $S$. pneumoniae IE affects middle-aged male patients, without previous pneumococcal vaccination (96\%), 54\% having at least two risk factors for IPD and $76 \%$ no risk factors for IE. Of note, almost $60 \%$ of the patients had had cardiac surgery in the week after IE diagnosis. The mortality rates at 90 days and 2 years after diagnosis were $17 \%$ and $33 \%$, respectively, although only $50 \%$ of deaths were directly related to IE.

Poor prognosis in our series is in accordance with another study showing that patients died mainly of causes directly related to IE such as heart failure (30\%), stroke (17\%), uncontrolled infection (14\%), and immediate post-surgical complication (10\%) [17]. In addition, mortality due to S. pneumoniae IE occurred early, in median 160 days [22-796] after diagnosis. Age $\geq$ 65 years was associated with pneumococcal IE mortality, and surgery with survival in our study and in another French study [7]. In our study, the subgroup of prosthetic valve pneumococcal IE had a mortality rate of $60 \%$ with only $20 \%$ of surgical procedures.

There is poor evidence regarding the usefulness of surgery in guidelines, although it has been suggested that surgery could play a protective role in pneumococcal IE [7]. In a study including 449 patients with left-sided IE of whom 240 (53\%) had had valve surgery, the 5year survival rate remained low with non-surgical treatment compared to early surgery (48\% vs $69.6 \% ; \mathrm{p}<0.001)$ [18]. In our study, mortality was significantly reduced in patients who underwent surgery. However, they were younger and had fewer comorbidities. Since the aortic valve is often damaged during pneumococcal IE, defining size, anatomy and calcification of the aortic valve, can be of help in surgical planning [16].

Antibiotic therapy has dramatically decreased IE mortality from $100 \%$ to $20-40 \%$ in comparison to the preantibiotic era [19]. Clear recommendations regarding antibiotherapy 
have been given by the ESC [16]. In our study, the type of antibiotics chosen was in accordance with ESC Guidelines for $74 \%$ of patients even though most patients were treated before 2015 ESC Guidelines publication [16]. Nevertheless, there are no differences between the 2009 and the 2015 ESC Guidelines for pneumococcus IE antimicrobial therapy. The appropriateness of combination therapy with gentamicin was difficult to estimate due to absence of susceptibility testing in patients' medical records. When used, gentamicin treatment was shorter than recommended.

In our cohort, only two patients were vaccinated with PPV23 before the occurrence of pneumococcal IE. In a Spanish study, vaccination data were available for only $12 \%$ ( $n=13 / 111)$ of the studied patients, of whom four had been vaccinated [3]. In another French study on a cohort of 30 patients in the 1990s, no patients received PPV23 before developing pneumococcal IE [7]. Since PPV23 uptake was recommended throughout our study period by the French health authorities, 39 patients (78\%) with predisposing IPD conditions should have received it. The two main reasons to explain low pneumococcal vaccine uptake are: (i) general practitioners could be less aware of vaccine recommendations that often change (20), (ii) a favorable opinion about vaccination in general is associated with higher vaccine uptake; the rate of vaccine hesitancy in France is one of the highest among European countries (21). However, PPV23 efficacy is $45-80 \%$ depending on study design (22). A randomized doubleblind study including 84496 adults aged $\geq 65$ years brought evidence that PCV13 could be more effective in this population preventing $75 \%$ of IPD (23). Robust data attesting to the possible positive impact of PCV13 alone or PCV13 followed two months later by PPV23 are still missing.

Our study had some limitations. Some data are missing due to the retrospective nature of the study. For instance, transesophageal echocardiogram was performed in only $70 \%$ of the patients. Thus, some local complications could have been missed. Our study was also not 
designed to compare pneumococcal IE to IE due to other bacteria. In addition, there is almost always survival bias in such a study: patients who live longer are more likely to receive treatment than patients who die early $(24,25)$. We could not perform the multivariate analysis planned in the protocol due to the small number of events. Although multicentric, our study may have underestimated the actual frequency of pneumococcal IE in France. Nonetheless, the calculated frequency of $1.3 \%$ seems to be in accordance with other studies: $0.86 \%$ in a Spanish cohort [3], 1\% and $1.6 \%$ in two French cohorts $(1,26)$. The missing MIC and antibiotic susceptibility testing, which were difficult to collect retrospectively, are important limitations in characterization of the medical strategy and comparison to 2015 ESC guidelines.

\section{Conclusion}

Although pneumococcal IE remains rare, its prognosis is poor within 90 days following diagnosis. Surgery seems to be of benefit for some patients. However, optimal timing of the procedure has yet to be determined. Robust data are still missing regarding the role of PCV13 combined to PPV23 vaccination in reducing pneumococcal IE incidence. 


\section{References}

1. Selton-Suty C, Célard M, Le Moing V, Doco-Lecompte T, Chirouze C, Iung B, et al. Preeminence of Staphylococcus aureus in Infective Endocarditis: A 1-Year Population-Based Survey. Clin Infect Dis. 2012 May 1;54(9):1230-9.

2. Hoen B. Évolution du profil des endocardites infectieuses : quoi de neuf ? Presse Médicale. 2010 Jun;39(6):701-3.

3. de Egea V, Muñoz P, Valerio M, de Alarcón A, Lepe JA, Miró JM, et al.

Characteristics and Outcome of Streptococcus pneumoniae Endocarditis in the XXI Century:

A Systematic Review of 111 Cases (2000-2013). Medicine (Baltimore). 2015

Sep;94(39):e1562.

4. Daudin M, Tattevin P, Lelong B, Flecher E, Lavoué S, Piau C, et al. Characteristics and prognosis of pneumococcal endocarditis: a case-control study. Clin Microbiol Infect Off Publ Eur Soc Clin Microbiol Infect Dis. 2016 Jun;22(6):572.e5-8.

5. Marrie TJ, Tyrrell GJ, Majumdar SR, Eurich DT. Risk factors for pneumococcal endocarditis. Eur J Clin Microbiol Infect Dis Off Publ Eur Soc Clin Microbiol. 2018 Feb;37(2):277-80.

6. Lindberg J, Prag J, Schønheyder HC. Pneumococcal endocarditis is not just a disease of the past: an analysis of 16 cases diagnosed in Denmark 1986-1997. Scand J Infect Dis. 1998;30(5):469-72.

7. Lefort A, Mainardi JL, Selton-Suty C, Casassus P, Guillevin L, Lortholary O. Streptococcus pneumoniae endocarditis in adults. A multicenter study in France in the era of penicillin resistance (1991-1998). The Pneumococcal Endocarditis Study Group. Medicine (Baltimore). 2000 Sep;79(5):327-37.

8. Martínez E, Miró JM, Almirante B, Aguado JM, Fernandez-Viladrich P, FernandezGuerrero ML, et al. Effect of penicillin resistance of Streptococcus pneumoniae on the presentation, prognosis, and treatment of pneumococcal endocarditis in adults. Clin Infect Dis Off Publ Infect Dis Soc Am. 2002 Jul 15;35(2):130-9.

9. Sands M, Brown RB, Ryczak M, Hamilton W. Streptococcus pneumoniae endocarditis. South Med J. 1987 Jun;80(6):780-2.

10. Centers for Disease Control and Prevention (CDC), Advisory Committee on Immunization Practices. Updated recommendations for prevention of invasive pneumococcal disease among adults using the 23-valent pneumococcal polysaccharide vaccine (PPSV23). MMWR Morb Mortal Wkly Rep. 2010 Sep 3;59(34):1102-6.

11. Brieu N, Varon E, Baraduc R, Brun M, Chardon H, Cremniter J, et al. Observatoires régionaux du pneumocoque : évolution de la résistance aux antibiotiques et des sérotypes de Streptococcus pneumoniae isolés en France entre 2009 et 2013. J Anti-Infect. 2015 Dec;17(4):145-50.

12. Braido F, Bellotti M, De Maria A, Cazzola M, Canonica GW. The role of Pneumococcal vaccine. Pulm Pharmacol Ther. 2008 Aug;21(4):608-15.

13. Borsu K, Krammisch H, Franckh M, Giet D. [Pneumococcal vaccine in adults: comparison of recommendations and discussion of its role]. Rev Med Liege. 2010 Apr;65(4):191-8.

14. Wintenberger C. [An update of Streptococcus pneumoniae: from genomic to clinic]. Med Mal Infect. 2010 Oct;40(10):605-9.

15. Prevention of pneumococcal disease: recommendations of the Advisory Committee on Immunization Practices (ACIP). MMWR Recomm Rep Morb Mortal Wkly Rep Recomm Rep. 1997 Apr 4;46(RR-8):1-24.

16. Habib G, Lancellotti P, Antunes MJ, Bongiorni MG, Casalta J-P, Del Zotti F, et al. 2015 ESC Guidelines for the management of infective endocarditis: The Task Force for the 
Management of Infective Endocarditis of the European Society of Cardiology (ESC). Endorsed by: European Association for Cardio-Thoracic Surgery (EACTS), the European Association of Nuclear Medicine (EANM). Eur Heart J. 2015 Nov 21;36(44):3075-128. 17. Fernandez-Hidalgo N, Almirante B, Tornos P, González-Alujas MT, Planes AM, Galiñanes M, et al. Immediate and long-term outcome of left-sided infective endocarditis. A 12-year prospective study from a contemporary cohort in a referral hospital. Clin Microbiol Infect. 2012 Dec;18(12):E522-30.

18. Bannay A, Hoen B, Duval X, Obadia J-F, Selton-Suty C, Le Moing V, et al. The impact of valve surgery on short- and long-term mortality in left-sided infective endocarditis: do differences in methodological approaches explain previous conflicting results? Eur Heart J. 2011 Aug;32(16):2003-15.

19. Lowes JA, Williams G, Tabaqchali S, Hill IM, Hamer J, Houang E, et al. 10 years of infective endocarditis at st. bartholomew's hospital: analysis of clinical features and treatment in relation to prognosis and mortality. The Lancet. 1980 Jan;315(8160):133-6.

20. Loubet P, Kernéis S, Groh M, Loulergue P, Blanche P, Verger P, et al. Attitude, knowledge and factors associated with influenza and pneumococcal vaccine uptake in a large cohort of patients with secondary immune deficiency. Vaccine. $2015 \mathrm{Jul}$ 17;33(31):3703-8.

21. Larson HJ, de Figueiredo A, Xiahong Z, Schulz WS, Verger P, Johnston IG, et al. The State of Vaccine Confidence 2016: Global Insights Through a 67-Country Survey. EBioMedicine. 2016 Oct;12:295-301.

22. Centers for Disease Control and Prevention (CDC), Advisory Committee on Immunization Practices. Updated recommendations for prevention of invasive pneumococcal disease among adults using the 23-valent pneumococcal polysaccharide vaccine (PPSV23). MMWR Morb Mortal Wkly Rep. 2010 Sep 3;59(34):1102-6.

23. Bonten MJM, Huijts SM, Bolkenbaas M, Webber C, Patterson S, Gault S, et al. Polysaccharide conjugate vaccine against pneumococcal pneumonia in adults. N Engl J Med. 2015 Mar 19;372(12):1114-25.

24. Tleyjeh IM, Baddour LM. The potential impact of survivor treatment selection bias on the perceived efficacy of valve surgery in the treatment of infective endocarditis. Clin Infect Dis Off Publ Infect Dis Soc Am. 2007 May 15;44(10):1392-3.

25. van Walraven C, Davis D, Forster AJ, Wells GA. Time-dependent bias was common in survival analyses published in leading clinical journals. J Clin Epidemiol. 2004 Jul;57(7):672-82.

26. Delahaye F, Goulet V, Lacassin F, Ecochard R, Selton-Suty C, Hoen B, et al. Characteristics of infective endocarditis in France in 1991. A 1-year survey. Eur Heart J. 1995 Mar;16(3):394-401. 
Table 1. Demographic and clinical characteristics of 50 patients with definite pneumococcal endocarditis

Characteristics*

$\mathbf{N}=\mathbf{5 0}$

\section{Baseline}

Age at diagnosis (years), mean \pm SD

$60 \pm 14$

Male gender

$38(76)$

IPD predisposing conditions

Asplenia

$>65$ years

Chronic pulmonary disease

$12(24)$

Repeted ENT infections

Cardiopathy

Alcoholism

Malignant blood disease

6

Renal failure

3

Kidney transplantation

3

IPD risk factors

none

1

$\geq 2$

27 (54)

No vaccine uptake before IE

IE predisposing conditions

Valvular prosthesis

5

Previous IE

1

Congenital cardiopathy

2

Valvular disease

$10(22)$

Intravenous drug use

1

Pacemaker

4

IE risk factors

$$
\begin{aligned}
& \text { none } \\
& 1 \\
& \geq 2
\end{aligned}
$$

6

6

\section{Clinical and biological manifestations}

Fever

Vascular phenomena**

Immunological phenomena***

0

Pneumonia

Meningitis

Arthritis

$10(20)$

Ocular infection

Other infectious localizations $\$$

Blood cultures positive for S. pneumoniae

Positive histopathological examination and/or culture of infected valve

8

Affected valve

Native 
Aortic

Tricuspid

\section{Transthoracic echocardiographic findings}

TTE positive for IE

Vegetations

Abscess

ENT: ear-nose-throat; IE: infective endocarditis; IPD: invasive pneumococcal disease; SD: standard deviation; TTE: transthoracic electrocardiography

* data are expressed in absolute number $(\%)$, otherwise indicated

** defined by the modified Dukes criteria as major arterial emboly, septic pulmonary infarct, infectious (mycotic) aneurysm, intracranial haemorrhage, conjunctival haemorrhage or Janeway's lesion.

***defined by the modified Dukes criteria as glomerulonephritis, Osler's nodes, Roth's spots or rheumatoid factor.

$\$$ sinusitis, muscular or gluteal abscess, acute otitis media, arteritis, stroke, infected aortic aneurysma, pericarditis. 
$\mathbf{N}=\mathbf{5 0}$

Time from $1^{\text {st }}$ symptoms onset to antibiotherapy, days (mean \pm SD)

$6.3 \pm 6.9$

Number of antibiotics, $n(\%)$

Monotherapy

Bitherapy

7

$\geq 3$ antibiotics

$28(56)$

$15(30)$

Antibiotic choice, $n(\%)$

$3^{\text {rd }}$ generation cephalosporin or amoxicillin

or penicillin $\mathrm{G}$ or vancomycin

Gentamicin

$50(100)$

$39(78)$

Antibiotic duration (mean \pm SD)

Total antibiotherapy duration, weeks

$5.5 \pm 2.3$

Beta-lactam or vancomycin duration, days

$37 \pm 14.7$

Gentamicin duration, days

$8.5 \pm 8.4$

Treatment in accordance with 2015 ESC Guidelines, n (\%)

Antibiotic choice

$37(74)$

Beta-lactam or vancomycin duration

$18(36)$

Gentamicin duration

2

Surgical procedure, $n(\%)$

Valvuloplasty

$28(56)$

Biological valve replacement

$15(30)$

Mechanical valve replacement

$10(20)$

Time from symptoms onset to surgery, days (median [ IQR 25-75])

$13.5[7.8-17.3]$

Time from diagnosis onset to surgery, days (median [ IQR 25-75])

$6.5[2.0-10.5]$

Pneumococcal vaccination after IE, $n(\%)$

$13(26)$

Death, $n(\%)$

$20(40)$

Delay from diagnosis to death, days (med [IQR 25-75])

160 [22-796]

Relapse, $n$

SD: standard deviation; IQR: interquartile range 
Figure legend

Figure 1. Kaplan Meier curves of survival probability after diagnosis for 50 patients with pneumococcal endocarditis.

Panel A: Survival probability at 90 days after diagnosis

Panel B: Survival probability at 2 years after diagnosis 
A

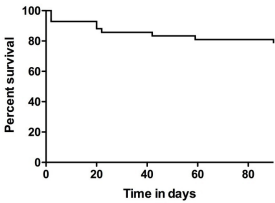

B

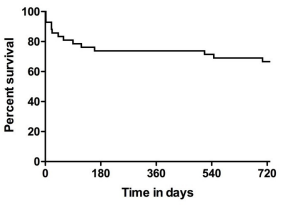

$\begin{array}{llllll}\text { At risk } & 50 & 31 & 31 & 30 & 28\end{array}$

